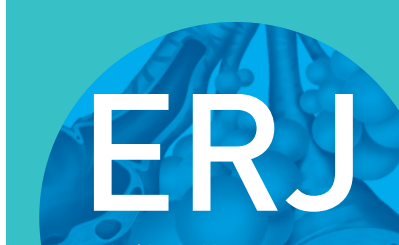

open research

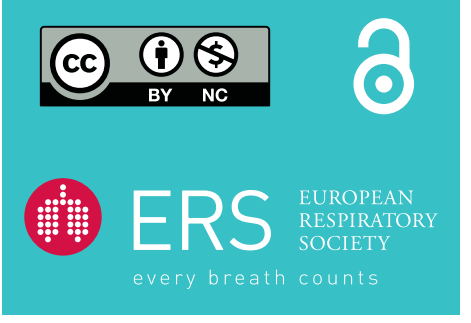

\section{De novo bronchiectasis in haematological malignancies: patient characteristics, risk factors and survival}

To the Editor:

Patients with haematological malignancies often develop secondary immunodeficiency and respiratory infections that could predispose towards bronchiectasis, and this is supported by case reports and a previous small case series of 22 patients [1-4]. To improve understanding of this association we performed a retrospective review of patients with clinically significant bronchiectasis (defined as radiological evidence of bronchiectasis plus chronic productive cough or recurrent respiratory tract infections requiring antibiotic therapy) and haematological malignancy attending our respiratory clinic at a UK teaching hospital between January 2014 and April 2018. Patients were investigated according to the British Thoracic Society guideline [5]. Bronchiectasis onset was defined as the computed tomography (CT) scan date showing bronchiectasis closest to when the clinical diagnosis was confirmed. $18.7 \%$ of patients were followed up for 1 year, $54.7 \%$ for 5 years, $20 \%$ for 10 years and $6.7 \%$ for $>10$ years. Data were extracted from electronic patient records under National Health Service research ethics committee approval 18/SC/ 0592 and analysed using SPSS version 22 (IBM, Chicago, IL, USA). For comparison between categorical variables, the Chi-squared or Fisher's exact tests were used as appropriate. Due to the nonparametric nature of the data, the Mann-Whitney test was used for the comparison of two continuous variables. Univariate analysis was used to identify factors associated with the number of lobes affected, IgG deficiency and mortality, and significant variables were entered alongside clinically relevant variables into a forward stepwise binary logistic regression model.

A total of 75 patients (median (interquartile range (IQR)) age 59 (27) years, 65.3\% male) with haematological malignancy and clinically significant bronchiectasis were identified. None of these patients had CT scan evidence of bronchiectasis at the time of haematological malignancy diagnosis. The most prevalent haematological malignancies were non-Hodgkin's lymphoma (NHL) (29.3\%), chronic lymphocytic leukaemia (CLL) (22.7\%), acute myeloid leukaemia (AML) (16\%) and Hodgkin's lymphoma (HL) (13.3\%). Of the probable causes of bronchiectasis, 45 patients (60\%) had had haematopoetic stem cell transplantation (HSCT), of which $37(82.2 \%)$ were allografts. 37 patients (50.7\%) were IgG deficient (IgG $<7 \mathrm{~g} \cdot \mathrm{L}^{-1}$, median (IQR) IgG for all patients $\left.6.5(6.5) \mathrm{g} \cdot \mathrm{L}^{-1}\right)$, and 20 patients $(26.7 \%)$ had both previous HSCT and IgG deficiency (figure 1a). For the remaining 13 patients, the bronchiectasis was suspected to be related to CLL $(n=5)$, intrapulmonary lymphoma $(n=2)$ or post-radiotherapy $(n=1)$, and unknown in five. All patients with AML, acute lymphocytic leukaemia (ALL), or multiple myeloma (MM) had had HSCT, compared to $60 \%$ of HL, $55.5 \%$ of NHL and $16.7 \%$ for CLL patients. 21 out of 45 (46.6\%) HSCT patients were diagnosed with pulmonary graft versus host disease (pGVHD) and these patients were younger than those without pGVHD (age 57 (19) versus 66 (23) years), $\mathrm{p}=0.015$ ). The median (IQR) time to development of bronchiectasis was 5 (7) years from diagnosis of haematological malignancy and developed faster in patients post-HSCT (3 (20) years, $14(31.1 \%)$ within 1 year) or treated with rituximab (4 (12) years, $14(40 \%)$ within 1 year) compared to all patients (5 (7) years, 10 (13.3\%) within 1 year). Most patients had cylindrical bronchiectasis (73 out of $75,97.3 \%$ ), with one having cystic bronchiectasis and another one varicose bronchiectasis. 40 patients (53.3\%) had bronchiectasis affecting three or more

@ERSpublications

Bronchiectasis occurs de novo in haematological malignancy and is associated with significant mortality and morbidity. Rituximab predisposes to IgG deficiency and survival time is significantly associated with age, $\mathrm{FEV}_{1} \%$ and stem cell transplantation. http://bit.ly/2KZwCZt

Cite this article as: José RJ, Hall J, Brown JS. De novo bronchiectasis in haematological malignancies: patient characteristics, risk factors and survival. ERJ Open Res 2019; 5: 00166-2019 [https://doi.org/10.1183/23120541.00166-2019].

Copyright $\odot$ ERS 2019. This article is open access and distributed under the terms of the Creative Commons Attribution NonCommercial Licence 4.0. 
a)
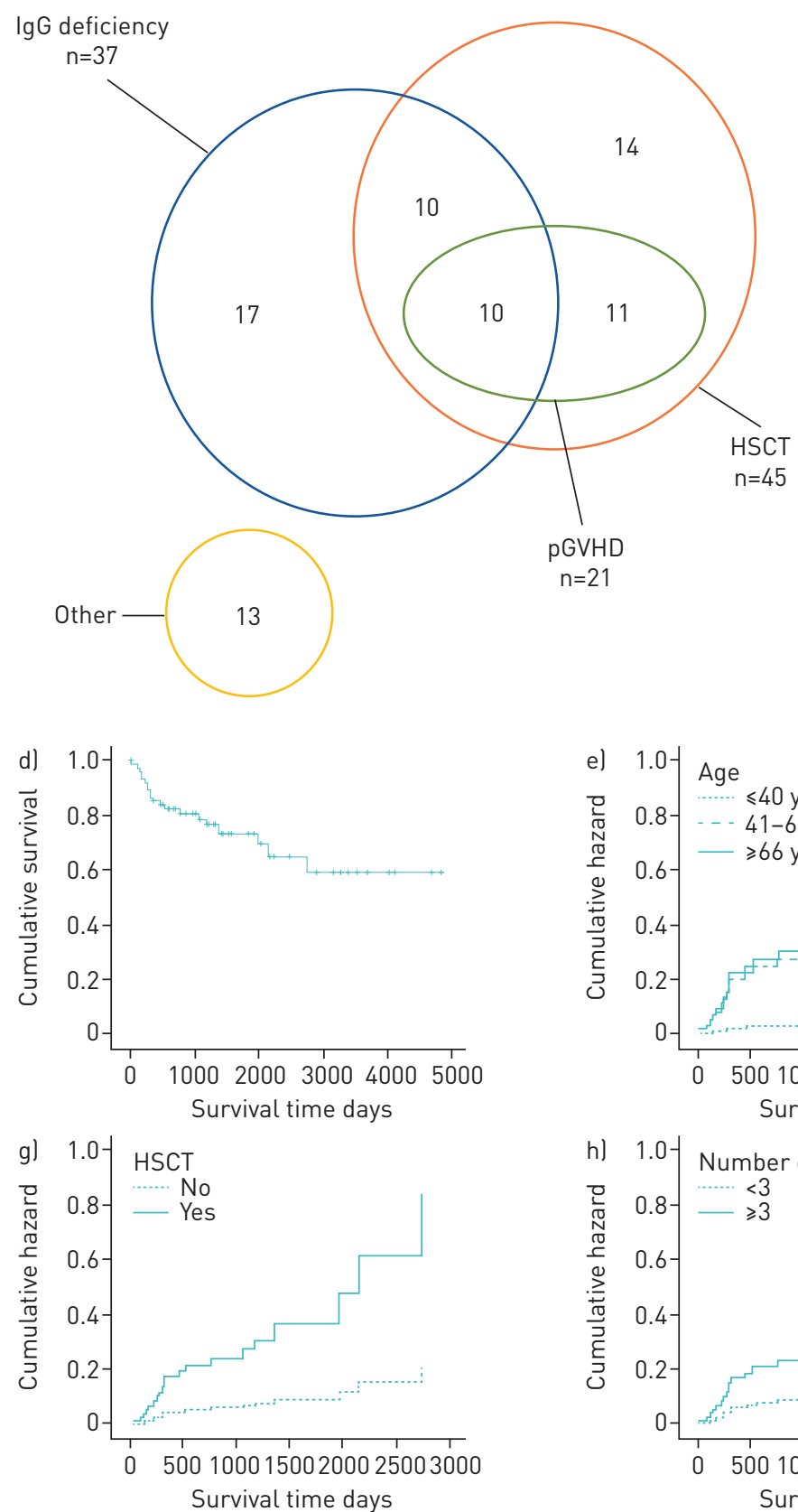
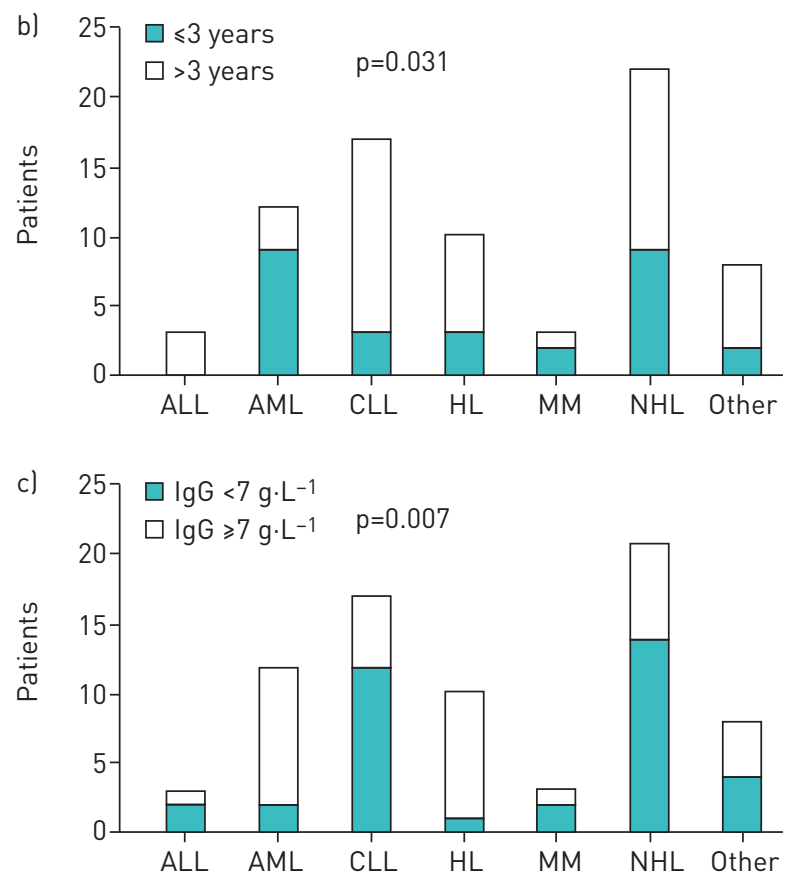

FIGURE 1 a) Potential aetiology of bronchiectasis in individuals with haematolgoical malignancy. b) Proportion of patients with onset of clinically significant bronchiectasis within 3 years of haematological diagnosis according to haematological diagnosis $(\mathrm{n}=75)$. c) Proportion of patients with IgG deficiency according to haematological diagnosis ( $n=73$ ). d) Kaplan-Meier survival analysis of patients with haematological malignancy from the time of bronchiectasis diagnosis (mean survival time of 3329 days (95\% Cl 2771-3888 days). Unadjusted hazard ratios (HRs) for e) age (age $\leqslant 40$ years: HR $0.104(95 \% \mathrm{Cl} 0.02-0.84), \mathrm{p}=0.034$; age $41-60$ years: HR 0.90 (95\% Cl 0.35-2.24), $\mathrm{p}=0.804)$ and f) forced expiratory volume in $1 \mathrm{~s}$ ( $\mathrm{FEV}_{1}$ ) (FEV 1 <40\% pred: HR 4.27 (95\% Cl 1.29-14.09), $\mathrm{p}=0.017$; $\mathrm{FEV}_{1} 41-80 \%$ pred: HR 2.12 (95\% Cl 0.71-6.34), $\left.\mathrm{p}=0.180\right)$. Age-adjusted HRs for g) haematological diagnosis for haematopoietic stem cell transplant (HSCT) (HR 3.94 (95\% Cl 1.03-15.14), $p=0.046$ ) and h) number of lobes affected (HR 2.70 (95\% Cl 1.00-7.34), p=0.051). pGVHD: pulmonary graft versus host disease; ALL: acute lymphocytic leukaemia; AML: acute myeloid leukaemia; CLL: chronic lymphocytic leukaemia; HL: Hodgkin's lymphoma; MM: multiple myeloma; NHL: non-Hodgkin's lymphoma.

lobes, and this was associated with HSCT (OR 4.00 (95\% CI 1.50-10.66), p=0.006) and pGVHD (OR 5.73 (95\% CI 1.70-19.31), $\mathrm{p}=0.005)$. In a binary logistic regression model including age, haematological malignancy, HSCT and pGVHD, only pGVHD maintained an association with three or more lobes affected (OR 6.7 (95\% CI 1.2-36.6), p=0.028). 
12 (16\%) patients were colonised with bacterial pathogens, including seven (9.3\%) with Pseudomonas aeruginosa. Eight colonised patients had IgG deficiency (21.6\%) versus three with normal IgG levels (8.3\%) $(\mathrm{p}=0.113)$ and the odds ratio for bacterial colonisation in those with IgG deficiency was 4.28 (95\% CI $0.89-20.49)(\mathrm{p}=0.069)$ after adjusting for rituximab treatment. 17 patients $(22.7 \%)$ had a past medical history of COPD or asthma. Smoking history was available for 73 patients $(92.3 \%)$, with $62 \%$ never smokers, 33\% ex-smokers and 5\% current smokers. Lung function values were available for 65 patients; mean $\pm \mathrm{SD}$ forced expiratory volume in $1 \mathrm{~s}\left(\mathrm{FEV}_{1}\right)$ and forced vital capacity $(\mathrm{FVC})$ were similar to that obtained by replacing missing values by multiple imputation $(74.9 \pm 24.8 \%$ versus $75.4 \pm 24.9 \%$ pred and $86.7 \pm 20.8 \%$ versus $87.7 \pm 21.6 \%$ pred, respectively). Airflow limitation ( $\mathrm{FEV}_{1}<80 \%$ pred) was present in $55 \%$ of patients, with patients with pGVHD having lower mean \pm SEM $\mathrm{FEV}_{1}$ and FVC $(61.3 \pm 6.6 \%$ versus $80.9 \pm 3.7 \%$ pred, $\mathrm{p}=0.003$; and $77.8 \pm 5.3 \%$ versus $91.7 \pm 3.5 \%$ pred, $\mathrm{p}=0.018$ ) and patients with bacterial colonisation having lower mean \pm SEM $\mathrm{FEV}_{1}(56.4 \pm 8.3 \%$ versus $79.1 \pm 3.8 \%$ pred, $\mathrm{p}=0.012)$.

IgG deficiency was associated with the type of haematological malignancy $(\mathrm{p}=0.007)$ and rituximab treatment; AML and HL patients were less likely (5.7\% versus $26.3 \%$ and $2.9 \%$ versus $22.5 \%$ respectively, $\mathrm{p}<0.05$ ) (figure 1c) and patients treated with rituximab were more likely (70.3\% versus $29.7 \%, \mathrm{p}<0.001$; OR 6.15 (95\% CI 2.23-16.95), $\mathrm{p}<0.001)$ to have IgG deficiency. $36(48 \%)$ patients had rituximab treatment, including all patients with CLL, $72.7 \%$ with NHL, $30 \%$ with $\mathrm{HL}$ and $10 \%$ with MM.

The mortality in the cohort was high at $26.7 \%$ (20 out of 75) with 1-, 5- and 10-year survival following diagnosis of bronchiectasis of $82 \%, 65 \%$ and $59 \%$ (figure $1 \mathrm{~d}$ ). Nonsurvivors had a median (IQR) survival of 324 (951) days, with the majority of patients post-HSCT dying within 2 years of the bronchiectasis diagnosis $(76.9 \%$ versus $42.9 \%$ of the non-HSCT patients, $\mathrm{p}=0.0001)$. In a binary logistic regression model including age, haematological diagnosis, HSCT, pGVHD, IgG deficiency, $\mathrm{FEV}_{1} \%$ pred and number of lobes affected, only age (OR 1.14 (95\% CI 1.03-1.27), p=0.016) and $\mathrm{FEV}_{1}$ (OR 0.91 (95\% CI 0.84-0.99, $\mathrm{p}=0.033$ ) were associated with overall mortality. Univariate analysis demonstrated that survival was improved in patients $\leqslant 40$ years old and reduced in patients with $\mathrm{FEV}_{1}<40 \%$ pred (figure $1 \mathrm{e}$ and f) [6]. In a Cox regression model including age, haematological malignancy, HSCT, pGVHD, bacterial colonisation, $\mathrm{FEV}_{1} \%$ pred and number of lobes, only age (hazard ratio (HR) 1.07 (95\% CI 1.03-1.12), p=0.001), $\mathrm{FEV}_{1}$ $\%$ pred (HR 0.96 (95\% CI 0.94-0.98), p<0.001) and number of lobes affected (HR 3.53 (95\% CI 1.27-9.81), $\mathrm{p}=0.016$ ) were significantly associated with survival duration (figure $1 \mathrm{~g}$ and $\mathrm{h}$ ).

This is the largest reported study of patients with bronchiectasis and haematological malignancy, and although retrospective, the data provide insight into the development of bronchiectasis in these patients. Our cohort had a male predominance, whereas non-CF bronchiectasis usually has a female predominance [7], probably reflecting that myeloid and lymphoid malignancies are more common in males [8]. Bronchiectasis in this cohort seemed to develop due to different mechanisms, either acquired IgG deficiency (mainly in patients with CLL or NHL) or allograft HSCT (47\% of whom had pGVHD compared to $<10 \%$ in all allograft HSCT patients) [9], but with some rarer causes. There was a strong association of rituximab treatment with IgG deficiency, and we suggest that there should be a high index of suspicion for bronchiectasis in patients with CLL and NHL who have received rituximab. Haematology patients frequently have multiple CT scans, allowing the timing of bronchiectasis development to be identified, and this showed the disease can develop surprisingly rapidly, frequently within a year in patients with IgG deficiency or after HSCT. Overall mortality was high, largely related to the high proportion of HSCT patients who died within 2 years of the diagnosis of bronchiectasis. Whether bronchiectasis increased mortality and was a cause of death in these patients or is just a marker for those with a poorer prognosis is not clear. Overall, our data suggest that patients with haematological malignancy can rapidly develop bronchiectasis, especially those receiving rituximab or allograft HSCT, and this disease may make a significant contribution to their morbidity and perhaps mortality.

Ricardo J. José ${ }^{1,2}$, Jocelin Hall ${ }^{1,2}$ and Jeremy S. Brown ${ }^{1,2}$

${ }^{1}$ Centre for Inflammation and Tissue Repair, UCL Respiratory, London, UK. ${ }^{2}$ Dept of Respiratory Medicine, University College London Hospital, London, UK.

Correspondence: Ricardo J. José, Centre for Inflammation and Tissue Repair, UCL Respiratory, 5 University Street, London, WC1E6JF, UK. E-mail: r.jose@ucl.ac.uk

Received: 01 July 2019 | Accepted: 19 Aug 2019

Conflict of interest: None declared. 
References

1 Morehead RS. Bronchiectasis in bone marrow transplantation. Thorax 1997; 52: 392-393.

2 Sargent MA, Cairns RA, Murdoch MJ, et al. Obstructive lung disease in children after allogeneic bone marrow transplantation: evaluation with high-resolution CT. AJR Am J Roentgenol 1995; 164: 693-696.

3 Graham NJ, Müller NL, Miller RR, et al. Intrathoracic complications following allogeneic bone marrow transplantation: CT findings. Radiology 1991; 181: 153-156.

4 Chen LW, McShane PJ, Karkowsky W, et al. De novo development of bronchiectasis in patients with hematologic malignancy. Chest 2017; 152: 683-685.

5 Pasteur MC, Bilton D, Hill AT. British Thoracic Society guideline for non-CF bronchiectasis. Thorax 2010; 65: Suppl. 1, i1-58.

6 Williams KM. How I treat bronchiolitis obliterans syndrome after hematopoietic stem cell transplantation. Blood 2017; 129: 448-455.

7 Finch S, Polverino E, Blasi F, et al. Sex differences in bronchiectasis patient characteristics: an analysis of the EMBARC cohort. Epidemiology 2018; 52: PA2282.

8 Smith A, Howell D, Patmore R, et al. Incidence of haematological malignancy by sub-type: a report from the Haematological Malignancy Research Network. Br J Cancer 2011; 105: 1684-1692.

9 Jose RJ, Faiz SA, Dickey BF, et al. Non-infectious respiratory disease in non-HIV immunocompromised patients. $\mathrm{Br}$ J Hosp Med (Lond) 2014; 75: 691-697. 